\title{
JNK1/2 expression and modulation of STAT3 signaling in oral cancer
}

\author{
IOANNIS GKOUVERIS ${ }^{1}$, NIKOLAOS NIKITAKIS ${ }^{1}$, MARIA KARANIKOU ${ }^{2}$, \\ GEORGE RASSIDAKIS $^{2}$ and ALEXANDRA SKLAVOUNOU ${ }^{1}$
}

\author{
${ }^{1}$ Department of Oral Pathology and Medicine, Dental School; ${ }^{2}$ First Department of Pathology, \\ Medical School, National and Kapodistrian University of Athens, 11527 Athens, Greece
}

Received May 28, 2015; Accepted April 22, 2016

DOI: $10.3892 / \mathrm{ol} .2016 .4614$

\begin{abstract}
Mitogen-activated protein kinases (MAPKs) are a family of protein kinases that link extracellular stimuli with intracellular responses and participate in numerous cellular processes such as growth, proliferation, differentiation, inflammation and apoptosis. Persistent activation of signal transducer and activator of transcription 3 (STAT3), which is accompanied by increases in STAT3 tyrosine phosphorylation, is associated with cell proliferation, differentiation and apoptosis in oral squamous cell carcinoma (OSCC). The role and significance of the activation of MAPKs, particularly of c-Jun N-terminal kinase (JNK), on STAT3 signaling in OSCC have not been thoroughly investigated. The present study examines the effects of JNK1/2 modulation on STAT3 signaling and cellular activities in OSCC cells. The expression levels of STAT3 [total, tyrosine phosphorylated (p-Tyr) and serine phosphorylated (p-Ser)], JNK, c-Jun and cyclin D1 were assessed in the OSCC cell lines SCC25 and SCC9. Inhibition of JNK1/2 was achieved by pharmacological agents (SP600125) and by small interfering RNA (siRNA) silencing, while JNK1/2 was induced by active MAPK kinase 7 . Cell proliferation and viability rates were also evaluated. Inhibition of JNK1/2 with either SP600125 treatment or specific siRNA silencing resulted in decreased levels of p-Ser STAT3 and increased levels of p-Tyr STAT3 and cyclin D1 in both cell lines. Furthermore, JNK1/2 inhibition resulted in a dose-dependent increase in cell growth and viability in both cell lines. Opposite results were observed with JNK1/2 induction in both cell lines. The present results are supportive of a potential tumor suppressive role of JNK1/2 signaling in OSCC, which may be mediated through negative crosstalk with the oncogenic STAT3 signaling pathway. The possible
\end{abstract}

Correspondence to: Dr Ioannis Gkouveris, Department of Oral Pathology and Medicine, Dental School, National and Kapodistrian University of Athens, Thivon 2 Street, 11527 Athens, Greece E-mail: igkouver@gmail.com

Key words: STAT3, JNK1/2, crosstalk, signaling, oral, squamous carcinoma therapeutic implications of JNK1/2 inhibition for patients with OSCC require to be investigated.

\section{Introduction}

Head and neck squamous cell carcinoma (HNSCC) is the sixth most common type of cancer, and is associated with a low 5-year survival rate, despite recent improvements in the understanding of its molecular basis (1). It is known that HNSCC development and progression depends on several aberrations in signaling molecular pathways, including epidermal growth factor receptor (EGFR), Ras, nuclear factor- $\kappa \mathrm{B}$, signal transducer and activator of transcription (STAT), Wnt/ $\beta$-catenin, transforming growth factor (TGF)- $\beta$ and phosphoinositide 3-kinase-AKT-mammalian target of rapamycin (mTOR) (2). Understanding the role of these pathways will provide valuable information on the mechanisms controlling oral cancer, thus providing clinically useful therapeutic targets.

STAT signaling has been involved in oncogenesis $(3,4)$. A number of previous findings strongly suggest that persistent activation of STAT3 in HNSCC, accompanied by increases in STAT3 tyrosine phosphorylation, is linked to cell proliferation, differentiation and apoptosis $(5,6)$. Aberrant activation of upstream signaling pathways, particularly TGF- $\alpha /$ EGFR, has been associated with constitutive activation of STAT3 molecules in HNSCC (3,7). STAT3 is also activated through other pathways such as the alpha-7-nicotinic receptor, interleukin (IL)-6, IL-10 and IL-22 receptor, and erythropoietin receptor pathways in several types of cells, including $\operatorname{HNSCC}(8,10)$. In general, ligand binding leads to the phosphorylation of tyrosine residues within the receptor, resulting in STAT3 tyrosine phosphorylation, dimer formation, nuclear translocation, DNA binding and transcriptional activity (3,11-13).

STATs could also become phosphorylated at the Ser727 residue in response to growth factor and cytokine stimulation (14). However, the role of STAT3 serine phosphorylation remains controversial (14). Previous studies have proposed a negative impact of Ser727 phosporylation on STAT3 activity $(13,15-17)$, while others have indicated that STAT3 serine phosphorylation by several kinases may upregulate the transcriptional activity and nuclear translocation of STAT3 in cancer $(18,19)$.

Mitogen-activated protein kinases (MAPKs) are a family of protein kinases that link extracellular stimuli with intracellular 
responses through phosphorylation of specific downstream target molecules in serine and threonine residues (20). MAPKs have been implicated in various cellular processes, including growth, proliferation, differentiation, inflammation and apoptosis $(21,22)$. In HNSCC, MAPKs constitute downstream targets of several molecules, including EGFR, Raf kinase and reactive oxygen species, whose aberrant regulation has been studied in this type of cancer $(21,23,24)$.

c-Jun N-terminal kinases (JNKs) are members of the MAPK family, and become activated by a stressful cellular environment, including ultraviolet irradiation, oxidative stress, changes in osmolarity or metabolism, DNA damage, heat shock and inflammatory cytokine signaling (25). JNK signaling has been studied in HNSCC with controversial findings; thus, while certain studies support a pro-oncogenic function of JNK, others provide evidence that JNKs act as tumor suppressors in HNSCC (26-28).

Previous studies have supported an association between the activation of specific members of the MAPK family and negative regulation of STAT3 signaling (29-34). For example, inhibition of STAT3 activities through extracellular signalregulated kinase (ERK) and p38-dependent pathways in human lung adenocarcinoma has been observed (35). Lim and Cao (15) have reported that activation of JNK by various stresses or by its upstream kinase MAPK kinase 7 (MKK7) may be linked to STAT3 inhibition, but the knowledge about the association between STAT3 and JNK remains insufficient and requires further investigation. Overall, there is evidence to suggest that MAPKs may modulate the activation status of STAT3 in various cell types, including cancer cells $(15,29-34)$. The role of MAPKs in cancer and STAT3 regulation may vary according to the type and status of the studied cells, indicating the requirement to investigate this association in a cell-specific manner.

The aim of the present study is to evaluate the activation status of JNK and STAT3 in HNSCC cell lines and to assess the effects of JNK modulation on STAT3 expression, as well as on cell proliferation and viability. Understanding the role of these molecules and their potential crosstalk in oral cancer cells may pave the way for the development of alternative treatment strategies for patients with HNSCC.

\section{Materials and methods}

Cell lines and cell culture. Experiments were performed using established cell lines of human OSCC (SCC9 and SCC25), which were obtained from the American Type Culture Collection (Manassas, VA, USA). Cells were cultured in a 1:1 mixture of Ham's F-12 and Dulbecco's modified Eagle's medium containing $10 \%$ fetal bovine serum, $100 \mathrm{U}$ penicillin and $400 \mathrm{ng} / \mathrm{ml}$ hydrocortisone (Sigma-Aldrich, St. Louis, $\mathrm{MO}, \mathrm{USA}$ ) at $37^{\circ} \mathrm{C}$ in a $5 \% \mathrm{CO}_{2}$ air atmosphere. Cells were subcultured by disaggregation with trypsin $(0.1 \%)$ and ethylenediaminetetraacetic acid $(0.01 \%)$ in phosphate-buffered saline (PBS) at $\mathrm{pH} 7.5$.

Selective inhibition of JNK1/2. Cells were plated in 6-well plates at a density of $5 \times 10^{4}$ cells/well and were allowed to grow to $80 \%$ confluency. Then, cells were either treated with vehicle alone [dimethyl sulfoxide (DMSO) at a maximum concentration of $0.1 \%$ ] or with the selective JNK $1 / 2$ inhibitor

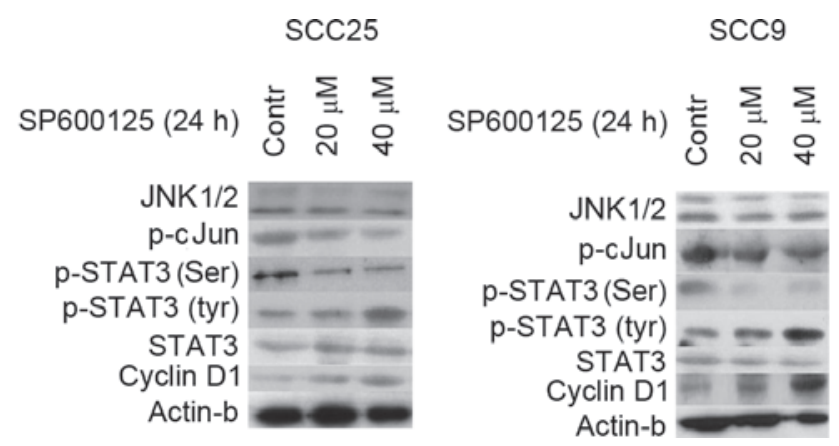

Figure 1. Effect of SP600125 inhibitor on STAT3 phosphorylation and cellular activity. Treatment of cells with SP600125 for 24 h had no effect on total JNK1/2 levels in the two cell lines evaluated, while it led to reduced levels of p-c-Jun in a dose-dependent manner. Selective inhibition of JNK1/2 also resulted in the downregulation of p-STAT3 (Ser727) and upregulation of p-STAT3 (Tyr705), which was most pronounced at the highest concentration tested in both cell lines. Cyclin D1 levels increased in both cell lines in a dose-dependent manner. Contr, control; STAT, signal transducer and activator of transcription; JNK, c-Jun N-terminal kinase; p-, phosphorylated.

SP600125 (Calbiochem; EMD Millipore, Billerica, MA, USA) at concentrations of $20-40 \mu \mathrm{M}$ for $24 \mathrm{~h}$.

Selective induction of JNK1/2 MAPK. Cells were plated in 6-well plates at a density of $2 \times 10^{5}$ cells/well and were allowed to grow to $80 \%$ confluency. Cells were then either treated with vehicle alone (DMSO at a maximum concentration of $0.1 \%$ ) or with the selective JNK1/2 inducer (active MKK7; Sigma-Aldrich) at concentrations of 5 and $10 \mu \mathrm{M}$ for $48 \mathrm{~h}$.

Small interfering RNA (siRNA) transfection. JNK1 and JNK2 siRNA and scrambled control siRNA were purchased from Qiagen, Inc. (Valencia, CA, USA). All siRNA transfections were performed using Lipofectamine 2000 (Invitrogen; Thermo Fisher Scientific, Inc., Waltham, MA, USA), according to the manufacturer's protocol, with a final siRNA concentration of 2 and $7 \mu \mathrm{M}$. OSCC cells were grown to mid-logarithmic phase and transiently transfected $\left(2 \times 10^{6}\right.$ cells $)$ with $50 \mu \mathrm{g}$ siRNA control or siRNA against JNK1/2 using Nucleofector ${ }^{\mathrm{TM}}$ reagent (Amaxa Biosystems; Lonza Group AG, Basel, Switzerland). Cells were collected at $48 \mathrm{~h}$ and whole lysates were analyzed by western blotting.

Western blot analysis. Cells were washed twice with ice-cold PBS, followed by lysis with radioimmunoprecipation assay buffer $(50 \mathrm{mM}$ Tris $\mathrm{pH} 7.4,150 \mathrm{mM} \mathrm{NaCl}, 1 \%$ Triton X-100, $1 \%$ deoxycholic acid sodium salt, $0.1 \%$ sodium dodecyl sulfate, $100 \mathrm{mg} / \mathrm{ml}$ phenylmethylsulfonyl fluoride, $1 \mathrm{mg} / \mathrm{ml}$ aprotinin, $1 \mathrm{mM}$ dichlorodiphenyltrichloroethane and $1 \mathrm{mM}$ sodium orthovanadate) for $10 \mathrm{~min}$ at $4^{\circ} \mathrm{C}$. The wells were scraped, and the recovered cell products were centrifuged at $40,000 \mathrm{x}$ g for $15 \mathrm{~min}$ at $4^{\circ} \mathrm{C}$. The concentration of the recovered proteins was measured and equalized using the Bio-Rad Protein Assay (Bio-Rad Laboratories, Inc., Hercules, CA, USA), according to the manufacturer's protocol.

Proteins in the total cell lysate were separated by SDS-PAGE (10\% separation gel and 5\% spacer gel) and electrotransferred to polyvinylidene difluoride films (BioRad Laboratories, Inc., Hercules, CA, USA). Blotted films were placed in blocking solution for $1 \mathrm{~h}$ at room temperature. 

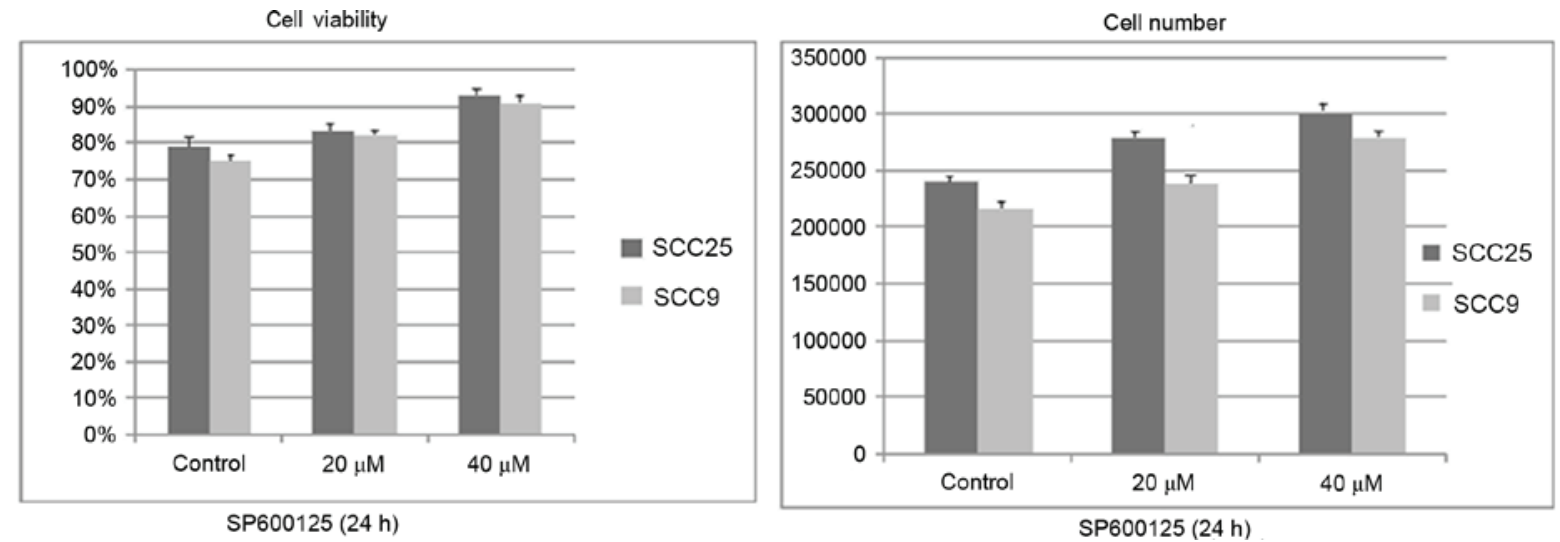

Figure 2. Effect of SP600125 on cell growth and viability. Treatment with SP600125 for 24 h resulted in a dose-dependent increase in cell growth (total number of cells) and cell viability (number of viable cells) in the two cell lines tested. The increase appears to be more prominent in the SCC25 cell line than in the SCC9 cell line. However, the observed changes did not reach statistical significance. Data are presented as the mean \pm standard deviation of four replicates.

$\operatorname{scC} 25$

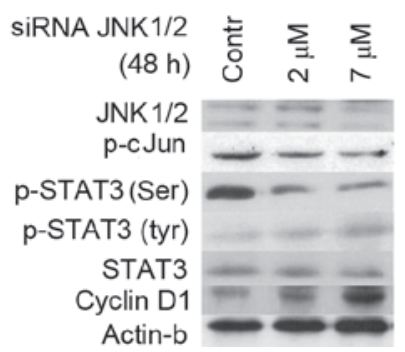

$\operatorname{scc} 9$

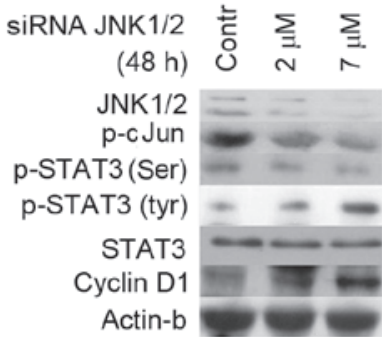

Figure 3. Effect of JNK1/2 silencing (via siRNA) on STAT3 phosphorylation and cellular activity. Following siRNA transfection ( $48 \mathrm{~h}$ ), western blotting revealed that the siJNK1/2 inhibited JNK total protein expression compared with control transfection experiments in the two cell lines tested. In addition, the levels of p-c-Jun were reduced in both cell lines in a dose-dependent manner. The decrease in JNK1/2 expression and c-Jun phosphorylation correlated with a decrease in p-STAT3 (Ser727) and an increase in p-Tyr STAT3 protein levels, which was most pronounced at the highest concentration assayed. Silencing of JNK1/2 was associated with markedly increased protein expression levels of cyclin D1 in both cell lines in a dose-dependent manner. Contr, control; STAT, signal transducer and activator of transcription; JNK, c-Jun N-terminal kinase; p-, phosphorylated; siRNA, small interfering RNA

Subsequently, they were probed with indicated primary antibodies overnight at $4^{\circ} \mathrm{C}$ (mouse monoclonal STAT3, diluted 1:250, cat no. 9139; rabbit polyclonal anti-phospho-STAT3 Tyr705, diluted 1:250, cat no. 9131; rabbit polyclonal phosphoSTAT3 Ser727, diluted 1:200, cat no. 9134; rabbit polyclonal anti-JNK1/2, diluted 1:250, cat no. 9252; rabbit polyclonal anti-p-c-jun ser63, diluted 1:200, cat no. 9261. and rabbit polyclonal cyclin-D1, diluted 1:250, cat no. 2922. All antibodies were purchased from Cell Signalling, Beverly, MA, USA. The film was washed thoroughly, incubated with goat polyclonal anti-rabbit IgG horse radish peroxidase secondary antibody (1:3.000; Santa Cruz Biotechnology, Santa Cruz, CA, USA, cat no. sc-2301) or anti-mouse IgG antibody (dilution, 1:3.000; Santa Cruz Biotechnology, cat no. sc-2031) with shaking at room temperature for $1 \mathrm{~h}$ at $25^{\circ} \mathrm{C} ; \beta$-actin was used as control (Santa Cruz Biotechnology, cat no. sc-47778). Proteins were visualized using an enhanced chemiluminescence

Cell proliferation and viability. Cells were counted with a Neubauer hemocytometer under an inverted microscope. Cell

viability upon treatment was determined by the Trypan blue dye exclusion test. All assays were performed in quadruplicate and the results are reported as the mean \pm standard deviation.

Statistical Analysis. Results of protein expression levels, cell viability and cell number of treated cells were compared with the results of untreated (control) cells respectively. Statistical analysis was performed using statistical Packages for the Social Sciences (SPSS) version 20. Paired groups were compared with the Student's t test and $\mathrm{P}<0.05$ was considered to indicate a statistically significant difference.

\section{Results}

Effects of SP600125 inhibitor on JNK1/2 and STAT3 protein expression and activation and cyclin Dl levels. The expression and activation status of JNK $1 / 2$ was examined in OSCC cells. According to the western blotting results, total protein levels of JNK1/2 were detected in the two OSCC cell lines tested (SCC9 and SCC25). Protein levels of p-c-Jun (immediate downstream of JNK), total STAT3, tyrosine phosphorylated (p-Tyr) STAT3, serine phosphorylated (p-Ser) STAT3 and cyclin D1 were also observed in both cell lines (Fig. 1).

The effectiveness of JNK1/2 inhibition was next assessed. Treatment of SCC25 cells with the JNK1/2 inhibitor SP600125 for $24 \mathrm{~h}$ resulted in a dose-dependent inhibition of c-Jun phosphorylation. Total JNK1/2 protein expression levels remained steady despite SP600125 treatment (Fig. 1).

Next, the effectiveness of JNK1/2 inhibition on STAT3 protein expression and activation was examined. In SCC25 cells, a significant reduction $(\mathrm{P}=0.03)$ of $\mathrm{p}$-Ser STAT3 could be detected following $24 \mathrm{~h}$ of SP600125 treatment in a dose-dependent manner. By contrast, an increase in the levels of p-Tyr STAT3 was observed, which was more pronounced at the highest concentration used $(40 \mu \mathrm{M})$. Treatment of SCC9 cells with SP600125 caused a decrease in p-Ser STAT3 only when a concentration of $40 \mu \mathrm{M}$ was used, along with a significant increase $(\mathrm{P}>0.05)$ in $\mathrm{p}$-Tyr STAT3 levels. In both cell lines, total STAT3 levels were not affected by SP600125 treatment (Fig. 1). 


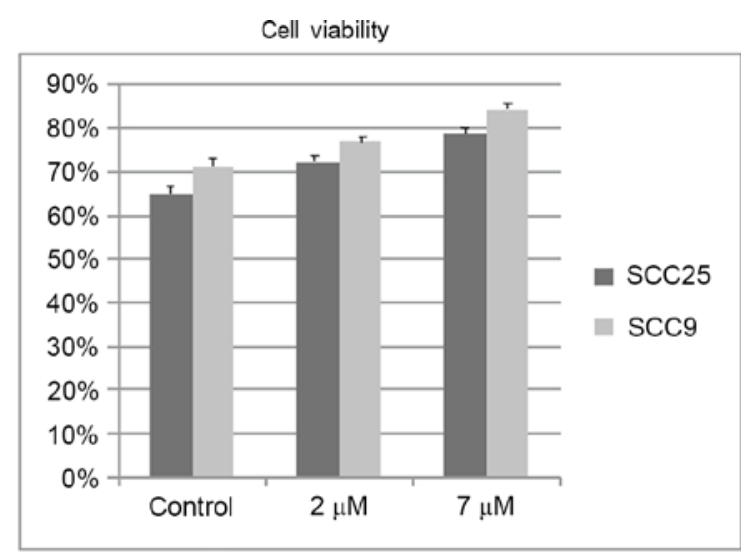

siRNA JNK1/2 (48 h)

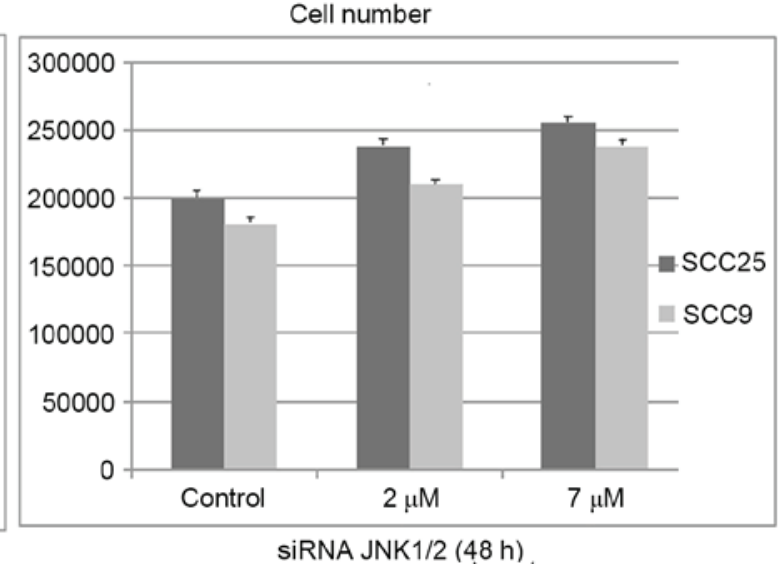

Figure 4. Effect of silencing JNK1/2 on cell growth and viability. Small interfering RNA treatment against JNK1/2 for 48 h resulted in a dose-dependent increase in cell growth and viability in the two cell lines tested, although it was not significant. Data are presented as the average \pm standard deviation of four replicates. JNK, c-Jun N-terminal kinase; siRNA, small interfering RNA.

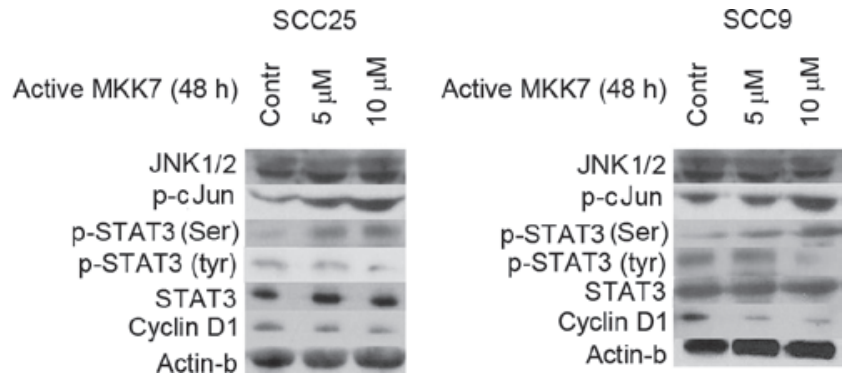

Figure 5. Effect of JNK1/2 induction on STAT3 phosphorylation and cellular activity. Induction of JNK1/2 with active MKK7 for $48 \mathrm{~h}$ enhanced the levels of p-c-Jun in a dose-dependent manner in the two cell lines tested without affecting the levels of total JNK1/2. Furthermore, induction of JNK1/2 resulted in the upregulation of p-STAT3 (Ser727) at both concentrations in SCC25 cells, and particularly at the highest concentration assayed in SCC9 cells. By contrast, the levels of p-Tyr STAT3 appeared to decrease upon treatment with active MKK7, particularly at the highest concentration used. Active MKK7 treatment of both cell lines for $48 \mathrm{~h}$ caused a downregulation in cyclin D1 expression levels in a dose-dependent manner. Contr, control; STAT, signal transducer and activator of transcription; JNK, c-Jun N-terminal kinase; p-, phosphorylated; siRNA, small interfering RNA; MKK7, mitogen-activated protein kinase kinase 7.

Western blotting demonstrated that inhibition of JNK1/2 was associated with increased expression levels of cyclin D1 in a dose-dependent manner. By contrast, the protein levels of actin remained stable throughout the treatment, indicating that the observed effects on the aforementioned proteins were not caused by a nonspecific reduction of protein expression (Fig. 1).

In summary, inhibition of JNK1/2 activity by SP600125 treatment was detected in both cell lines and was associated with increased levels of p-Tyr STAT3 and cyclin D1, and decreased levels of p-Ser STAT3, particularly at the highest concentration used.

Effects of SP600125 inhibitor on cell growth and viability. Treatment with SP600125 for 24 h resulted in a dose-dependent increase in cell growth (total number of cells) and cell viability (number of viable cells) in the two cell lines tested. The increase appeared to be more prominent in the SCC25 cell line than in the SCC9 cell lines. However, the observed changes were not significant (Fig. 2).
Effects of JNK1/2 siRNA silencing on JNK and STAT3 protein expression and activation and cyclin D1 levels. In order to corroborate the results from the pharmacological inhibition of JNK1/2, specific inhibition was performed by siRNA-targeting of JNK1/2 in SCC25 and SCC9 cell lines. Following $48 \mathrm{~h}$ of transfection with a specific siRNA against JNK1/2, western blot analysis revealed that JNK1/2 was efficiently silenced, which was accompanied by a dose-dependent decrease in the protein levels of p-c-Jun compared with the control in both cell lines (Fig. 3).

Decreases in JNK1/2 protein expression and c-Jun phosphorylation following $48 \mathrm{~h}$ of treatment with 2 and $7 \mu \mathrm{M}$ specific siRNA against JNK1/2 correlated with a decrease in the levels of p-Ser STAT3 in both cell lines. More prominent was this decrease at higher concentration $(7 \mu \mathrm{M})$. Regarding STAT3 tyrosine phosphorylation, an upregulation in the levels of p-Tyr STAT3 was detected, particularly at the highest concentration tested. Total STAT3 protein levels remained steady in both cell lines (Fig. 3).

Furthermore, western blot analysis demonstrated that silencing of JNK1/2 was associated with markedly increased levels of cyclin D1 protein expression in a dose-dependent manner in both cell lines, while the protein levels of $\beta$-actin remained stable (Fig. 3).

In summary, specific silencing of JNK $1 / 2$ resulted in decreases in p-Ser STAT3 and cyclin D1 levels and increases in p-Tyr-STAT3 levels in both cell lines.

Effects of silencing JNK1/2 on cell growth and viability. Similar to the effects of the chemical inhibitor SP600125, siRNA treatment against JNK1/2 for $48 \mathrm{~h}$ resulted in a dose-dependent increase in cell growth and viability in both cell lines, although it was not significant (Fig. 4).

Effects of JNK1/2 induction on JNK1/2 and STAT3 protein expression and activation and cyclin Dl levels. To further investigate the significance of JNK $1 / 2$ in the modulation of STAT3, pharmacological induction of JNK/2 was performed using active MKK7 to further induce JNK1/2 expression. Treatment of cells with selective MKK7 inducer efficiently upregulated the levels of p-c-Jun in a dose-dependent manner 
Cell viability

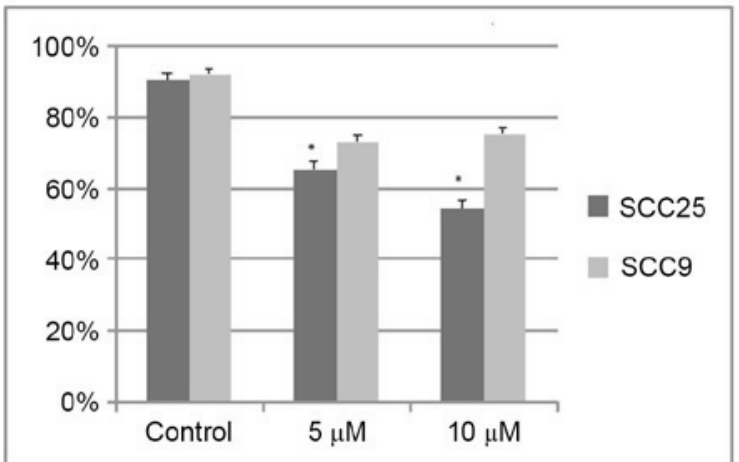

Active MKK7 $(48 \mathrm{~h})$
Cell number

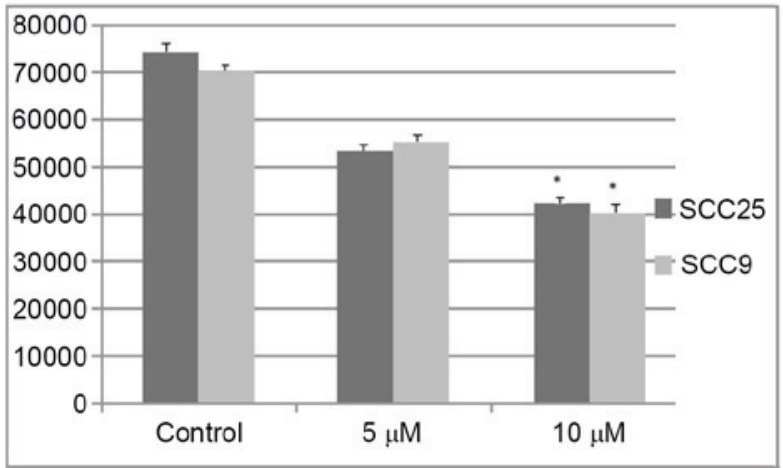

Active MKK7 (48 h)

Figure 6. Effect of c-Jun N-terminal kinase 1/2 induction on cell growth and viability. Active MKK7 treatment at the highest concentration tested for 48 h resulted in a significant dose-dependent decrease in cell growth and viability, which were more prominent in SCC25 than in SCC9 cells. Cell growth changes were significant at the highest concentration tested, compared with the control, in both cell lines $(\mathrm{SCC} 25 \mathrm{P}=0.039$; $\mathrm{SCC} 9 \mathrm{P}=0.044)$, while cell viability changes were significant at both concentrations, compared with the control, in the SCC25 cell line $(5 \mu \mathrm{M} \mathrm{P}=0.041,10 \mu \mathrm{M} \mathrm{P}=0.03)$. Data are presented as the average \pm standard deviation of four replicates. MKK7, mitogen-activated protein kinase kinase 7.

in both cell lines without affecting the total JNK1/2 levels (Fig. 5).

Treatment of both cell lines with JNK1/2 inducer for $48 \mathrm{~h}$ resulted in induced phosphorylation of STAT3 at Ser727 at the two concentrations tested in SCC25 cells, and particularly at the highest concentration assayed in SCC9 cells. By contrast, p-Tyr STAT3 levels appeared to decrease following treatment with active MKK7, particularly at the highest concentration tested. Total STAT3 levels were not affected by JNK1/2 induction in either cell line (Fig. 5).

With regards to cyclin D1, active MKK7 treatment of both cell lines for $48 \mathrm{~h}$ caused a downregulation in cyclin D1 expression levels in a dose-dependent manner. The levels of $\beta$-actin protein remained stable despite the treatment (Fig. 5).

In summary, JNK/2 induction caused an upregulation of p-Ser STAT3 levels in the two cell lines analyzed, as well as decreases in the levels of p-Tyr-STAT3 and cyclin D1.

Effects of JNK1/2 induction on cell growth and viability. Active MKK7 treatment at the highest concentration tested for $48 \mathrm{~h}$ resulted in a significant dose-dependent decrease in cell growth and viability, which were more prominent in the SCC25 cell line than in the SCC9 cell line. Cell growth changes were significant at the highest concentration assayed in both cell lines (SCC25 P=0.039; SCC9 P=0.044), compared with the control, while cell viability changes were significant at the two concentrations tested in the SCC25 cell line, compared with the control $(5 \mu \mathrm{M} \mathrm{P}=0.041 ; 10 \mu \mathrm{M}$ $\mathrm{P}=0.03$ (Fig. 6).

\section{Discussion}

The role of JNKs in cancer development is still under investigation. Activation of the JNK signaling pathway in response to several extracellular stimuli has been viewed as a critical event that leads to apoptotic or non-apoptotic cell death in several cells, including bronchial epithelial, human hepatoma, hepatocellular carcinoma and osteosarcoma cells (36-39). By contrast, JNK has also been reported to promote cell transformation and proliferation in cancer stem cells (40). Recent data suggest that the role of JNK1 and JNK2 in cancer is diverse, resulting in either promotion or suppression of tumor formation or size in different types of cancer, which emphasizes the significance of understanding the dual role of JNKs and the molecular background of these distinct functions in different tumors (41). For example, Seki et al (42) highlighted the relevant role of JNK signaling in the initiation and progression of liver cancer, and Leventaki et al (43) reported that JNK activation induces tumor cell proliferation in classical Hodgkin lymphoma. Jia et al (44) reported that the activation of JNK contributes to dihydroartemisinin-induced autophagy in pancreatic cancer cells, and Shi et al (45) suggested that JNK enhances the tumor suppressive role of p53 and promotes apoptosis in several cell cancer lines, including colon, breast carcinoma and osteosarcoma.

The functional role of JNK signaling has also been studied in HNSCC, with conflicting findings. Gross et al (27) observed that inhibition of c-Jun with SP600125 suppresses the growth of HNSCC cells. In addition, Yunoki et al (28) proposed that combined silencing of B-cell lymphoma 2-associated athanogene 3 (a co-chaperone of heat shock protein 70) and inhibition of the JNK signaling pathway may be an option in hyperthermia therapy in OSCC. However, several studies highlighted the role of activated JNK in apoptosis and tumor suppression in HNSCC. For example, Boivin et al (26) demonstrated that JNK mediates radiotherapy-induced apoptosis in human HNSCC cell lines, and Chen et al (46) demonstrated that the apoptotic effect of cisplatin and cordycepin act synergistically through the activation of the JNK/caspase-7/poly (ADP-ribose) polymerase signaling pathway in the human oral cancer cell line OC3. Furthermore, Noutomi et al (47) reported that JNK activation is involved in the molecular mechanism of tumor necrosis factor-related apoptosis-inducing ligand-induced cell death in HNSCC.

The present results appear to support a rather onco-suppressive role of JNK in oral cancer, since JNK1/2 inhibition was associated with a noticeable (although not significant) increase in the number of living OSCC cells in a 
dose-dependent manner, which was accompanied by a corresponding upregulation in the levels of cyclin D1. Previous studies examined the role of JNK in mediating the apoptotic effect of several pharmacological agents or anticancer drugs in HNSCC cell lines, including N-(4-hydroxyphenyl) retinamide (4HPR), pepsin-digested bovine lactoferrin, 5-aminolevulinic acid,MLN4924, 6-(N,N -dimethylamino)-2-(naphthalene-1-yl) -4-quinazolinone, fomitoside-K, mevastatin and AZD8055 (an mTOR inhibitor), and their biological mechanisms of apoptosis (48-55). Consistent with the present results, an antitumor role of JNK has been proposed upon treatment of HNSCC cells with the JNK inhibitor SP600125, which diminished the aforementioned pharmacological agents-induced apoptosis (47-54). Similarly, Li and Johnson (56) demonstrated that pharmacological inhibition of JNK with SP600125 markedly inhibited bortezomib-induction of autophagy regulatory proteins and autophagosome formation in HNSCC.

In the present study, selective siRNA-mediated JNK inhibition induced similar results to those of JNK pharmacological inhibition in a dose-dependent manner in the two cell lines evaluated, followed by a non-significant increase in the total number of cells and cell viability levels. Using similar siRNA techniques, Kim et al (49) demonstrated that suppression of JNK1 and JNK2 decreased, whereas overexpression of wild-type JNK1 enhanced, 4HPR-induced apoptosis. In addition, Li et al (48) described that JNK inhibition by RNA interference alleviated AZD8055-induced cell death in HNSCC.

By contrast, the present study has demonstrated that pharmacological induction of JNK appears to have the opposite effects to those aforementioned in the pharmacological and selective siRNA-mediated JNK inhibition. Notably, the decrease in the total cell number follows the relative decrease in cyclin D1 levels. In agreement with these results, Schramek et al (57) indicated that MKK7 functions as a major tumor suppressor in lung and mammary cancer in mice, while Tang et al (37) reported that alpinetin decreases proliferation of human hepatoma cells through the activation of MKK7, and Dai et al (58) reported MKK7/JNK1-dependent apoptosis in human acute myeloid leukemia cells.

Regarding a potential crosstalk between STAT3 and JNK, constitutive activation of STAT3, accompanied by increases in STAT3 tyrosine phosphorylation, is known to regulate cell proliferation, differentiation and apoptosis in various types of cancer $(5,6)$. However, the role of serine phosphorylation is less well understood. Although there is significant evidence in favor of the important role of STAT3 in head and neck cancer, the mechanism of STAT3 hyperactivation in this type of cancer remains to be completely understood (59). STAT proteins are important downstream targets of MAPKs, which are involved in the regulation of STAT proteins through crosstalk signaling (12).

The role of specific MAPKs, including JNK, in STAT3 regulation is not completely understood. In a previous study (60), the present authors investigated the role of ERK1/2, and demonstrated that ERK induced the upregulation of STAT3 Ser727 phosphorylation, while phosphorylation of Tyr705 did not appear to experience major changes.

The present study focused on the role of JNK in the regulation of STAT3 signaling, and investigated whether changes in the expression and activation status of JNK $1 / 2$ affect STAT3 tyrosine and/or serine phosphorylation and the total protein expression levels of STAT3 in HNSCC cell lines. Chemical inhibition (via SP600125) or selective targeting (via siRNA) of the MAPK JNK1/2 downregulated STAT3 serine phosphorylation, which was accompanied by a moderate increase in p-Tyr STAT3 levels. Induction of JNK had the opposite effects, resulting in upregulation of STAT3 serine phosphorylation and downregulation of STAT3 tyrosine phosphorylation.

Crosstalk between JNK and STATs has been described in several cases. For example, Kim et al (61) indicated that the development of doxorubicin resistance in cancer cell lines is correlated with the activation of STAT3 through the JNK signaling pathway. In addition, Guo et al (62) observed that JNK inhibition leads to reduced STAT3 Ser727 phosphorylation in breast cancer cells, and noticed that the antitumor activity of oncrasin-72 was followed by JNK activation and inhibition of Janus kinase 2/STAT3 phosphorylation. Similar results were obtained in previous studies in human bronchial epithelial cells, where JNK inhibition through SP600125 or JNK silencing reduced Ser727 phosphorylation of STAT3 $(39,63)$. Additionally, Lim and Cao (15) noticed that STAT3 is negatively regulated through JNK-induced serine phosphorylation by JNK in monkey kidney cells. Shirakawa et al (64) studied STAT6, another member of the STAT family, and suggested that JNK phosphorylates Ser707 of STAT6 and leads to deactivation and inhibition of the transcription of STAT6-responsive genes in human HeLa and HEK293 cells.

In summary, the present findings support a potential onco-suppressive role of JNK1/2 in OSCC, as the activation of JNK appears to downregulate cell proliferation and viability, and decreases cyclin D1 expression levels. It appears that oncogenic STAT3 constitutive signaling in OSCC cells is negatively regulated by JNK. The JNK-STAT3 crosstalk is mediated mostly through JNK-induced upregulation of STAT3 phosphorylation at Ser727, and downregulation of STAT3 phosphorylation at Tyr705, which collectively may cause the inhibition of STAT3 activity.

It is possible that the role of JNK in STAT3 modulation varies according to the type and status of the studied cells, indicating the requirement for identifying the role of MAPK activation in relation to STAT3 signaling in specific cell types. Understanding the complexity of the MAPK signaling pathway and the crosstalk between other major molecules such as STAT3 will be helpful to design pharmacological therapies for cancer prevention.

In conclusion, the present results indicate that JNK may be a potential significant molecular target of novel anticancer therapies for patients with OSCC.

\section{Acknowledgements}

The present study was co-financed by the European Union [Brussels, Belgium; European Social Fund (ESF), and Greek national funds through the Operational Program 'Education and Lifelong Learning' of the National Strategic Reference Framework-Research Funding Program 'Heracleitus II. Investing in knowledge society through the ESF' (Athens, Greece) grant no. 388/1/359. 


\section{References}

1. Chin D, Boyle GM, Porceddu S, Theile DR, Parsons PG and Coman WB: Head and neck cancer: Past, present and future. Expert Rev Anticancer Ther 6: 1111-1118, 2006.

2. Molinolo AA, Amornphimoltham P, Squarize CH, Castilho RM, Patel V and Gutkind JS: Dysregulated molecular networks in head and neck carcinogenesis. Oral Oncol 45: 324-334, 2009.

3. Rane SG and Reddy EP: Janus kinases: Components of multiple signaling pathways. Oncogene 19: 5662-5679, 2000.

4. Song JI and Grandis JR: STAT signaling in head and neck cancer. Oncogene 19: 2489-2495, 2000.

5. Kijima T, Niwa H, Steinman RA, Drenning SD, Gooding WE, Wentzel AL, Xi S and Grandis JR: STAT3 activation abrogates growth factor dependence and contributes to head and neck squamous cell carcinoma tumor growth in vivo. Cell Growth Differ 13: 355-362, 2002.

6. Leeman RJ, Lui VW and Grandis JR: STAT3 as a therapeutic target in head and neck cancer. Expert Opin Biol Ther 6: 231-241, 2006.

7. Bromberg J: Stat proteins and oncogenesis. J Clin Invest 109: 1139-1142, 2002

8. Jewett A, Head C and Cacalano NA: Emerging mechanisms of immunosuppression in oral cancers. J Dent Res 85: 1061-1073, 2006

9. Lai SY and Johnson FM: Defining the role of the JAK-STAT pathway in head and neck and thoracic malignancies: Implications for future therapeutic approaches. Drug Resist Updat 13 67-78, 2010.

10. Naher L, Kiyoshima T, Kobayashi I, Wada H, Nagata K, Fujiwara H, Ookuma YF, Ozeki S, Nakamura S and Sakai H: STAT3 signal transduction through interleukin-22 in oral squamous cell carcinoma. Int J Oncol 41: 1577-1586, 2012.

11. Bromberg J and Darnell JE Jr: The role of STATs in transcriptional control and their impact on cellular function. Oncogene 19: 2468-2473, 2000.

12. Decker T and Kovarik P: Serine phosphorylation of STATs Oncogene 19: 2628-2637, 2000.

13. Chung J, Uchida E, Grammer TC and Blenis J: STAT3 serine phosphorylation by ERK-dependent and -independent pathways negatively modulates its tyrosine phosphorylation. Mol Cell Biol 17: 6508-6516, 1997.

14. Reich NC and Liu L: Tracking STAT nuclear traffic. Nat Rev Immunol 6: 602-612, 2006.

15. Lim CP and Cao X: Serine phosphorylation and negative regulation of Stat3 by JNK. J Biol Chem 274: 31055-31061, 1999.

16. Venkatasubbarao K, Choudary A and Freeman JW: Farnesyl transferase inhibitor (R115777)-induced inhibition of STAT3 (Tyr705) phosphorylation in human pancreatic cancer cell lines require extracellular signal-regulated kinases. Cancer Res 65 : $2861-2871,2005$

17. Wakahara R, Kunimoto $H$, Tanino $K$, Kojima $H$, Inoue $A$ Shintaku H and Nakajima K: Phospho-Ser727 of STAT3 regulates STAT3 activity by enhancing dephosphorylation of phospho-Tyr705 largely through TC45. Genes Cells 17: 132-145, 2012 .

18. Sakaguchi M, Oka M, Iwasaki T, Fukami Y and Nishigori C: Role and regulation of STAT3 phosphorylation at Ser727 in melanocytes and melanoma cells. J Invest Dermatol 132: $1877-1885,2012$.

19. Aggarwal BB, Kunnumakkara AB, Harikumar KB, Gupta SR, Tharakan ST, Koca C, Dey S and Sung B: Signal transducer and activator of transcription-3, inflammation, and cancer: How intimate is the relationship? Ann N Y Acad Sci 1171: 59-76, 2009

20. Johnson GL and Lapadat R: Mitogen-activated protein kinase pathways mediated by ERK, JNK, and p38 protein kinases. Science 298: 1911-1912, 2002.

21. Maggioni D, Gaini R, Nicolini G, Tredici G and Garavello W: MAPKs activation in head and neck squamous cell carcinomas. Oncol Rev 5: 223-231, 2011.

22. Kim TW, Michniewicz M, Bergmann DC and Wang ZY: Brassinosteroid regulates stomatal development by GSK3-mediated inhibition of a MAPK pathway. Nature 482: 419-422, 2012.

23. Kim JY, An JM, Chung WY, Park KK, Hwang JK, Kim DS Seo SR and Seo JT: Xanthorrhizol induces apoptosis through ROS-mediated MAPK activation in human oral squamous cell carcinoma cells and inhibits DMBA-induced oral carcinogenesis in hamsters. Phytother Res 27: 493-498, 2013.
24. Li B, Lu L, Zhong M, Tan XX, Liu CY, Guo Y and Yi X: Terbinafine inhibits KSR1 and suppresses Raf-MEK-ERK signaling in oral squamous cell carcinoma cells. Neoplasma 60: 406-412, 2013.

25. Wada T and Penninger JM: Mitogen-activated protein kinases in apoptosis regulation. Oncogene 23: 2838-2849, 2004.

26. Boivin A, Hanot M, Malesys C, Maalouf M, Rousson R, Rodriguez-Lafrasse $\mathrm{C}$ and Ardail D: Transient alteration of cellular redox buffering before irradiation triggers apoptosis in head and neck carcinoma stem and non-stem cells. PLoS One 6: e14558, 2011.

27. Gross ND, Boyle JO, Du B, Kekatpure VD, Lantowski A, Thaler HT, Weksler BB, Subbaramaiah K and Dannenberg AJ: Inhibition of Jun NH2-terminal kinases suppresses the growth of experimental head and neck squamous cell carcinoma. Clin Cancer Res 13: 5910-5917, 2007.

28. Yunoki T, Kariya A, Kondo T, Hayashi A and Tabuchi Y: The combination of silencing BAG3 and inhibition of the JNK pathway enhances hyperthermia sensitivity in human oral squamous cell carcinoma cells. Cancer Lett 335: 52-57, 2013.

29. Ahmed ST, Mayer A, Ji JD and Ivashkiv LB: Inhibition of IL-6 signaling by a p38-dependent pathway occurs in the absence of new protein synthesis. J Leukoc Biol 72: 154-162, 2002.

30. Jain N, Zhang T, Fong SL, Lim CP and Cao X: Repression of Stat 3 activity by activation of mitogen-activated protein kinase (MAPK). Oncogene 17: 3157-3167, 1998.

31. Quadros MR, Peruzzi F, Kari C and Rodeck U: Complex regulation of signal transducers and activators of transcription 3 activation in normal and malignant keratinocytes. Cancer Res 64: 3934-3939, 2004.

32. Tkach M, Rosemblit C, Rivas MA, Proietti CJ, Díaz Flaqué MC, Mercogliano MF, Beguelin W, Maronna E, Guzmán P, Gercovich FG, et al: p42/p44 MAPK-mediated Stat3Ser727 phosphorylation is required for progestin-induced full activation of Stat3 and breast cancer growth. Endocr Relat Cancer 20: 197-212, 2013.

33. Sengupta TK, Talbot ES, Scherle PA and Ivashkiv LB: Rapid inhibition of interleukin-6 signaling and Stat3 activation mediated by mitogen-activated protein kinases. Proc Natl Acad Sci USA 95: 11107-11112, 1998.

34. Kovarik P, Stoiber D, Eyers PA, Menghini R, Neininger A, Gaestel M, Cohen P and Decker T: Stress-induced phosphorylation of STAT1 at Ser727 requires p38 mitogen-activated protein kinase whereas IFN-gamma uses a different signaling pathway. Proc Natl Acad Sci USA 96: 13956-13961, 1999.

35. Xue P, Zhao Y, Liu Y, Yuan Q, Xiong C and Ruan J: A novel compound RY10-4 induces apoptosis and inhibits invasion via inhibiting STAT3 through ERK-, p38-dependent pathways in human lung adenocarcinoma A549 cells. Chem Biol Interact 209: 25-34, 2014.

36. Song IS, Jun SY, Na HJ, Kim HT, Jung SY, Ha GH, Park YH, Long LZ, Yu DY, Kim JM, et al: Inhibition of MKK7-JNK by the TOR signaling pathway regulator-like protein contributes to resistance of HCC cells to TRAIL-induced apoptosis. Gastroenterology 143: 1341-1351, 2012.

37. Tang RX, Kong FY, Fan BF, Liu XM, You HJ, Zhang P and Zheng KY: HBx activates FasL and mediates HepG2 cell apoptosis through MLK3-MKK7-JNKs signal module. World J Gastroenterol 18: 1485-1495, 2012.

38. Sau A, Filomeni G, Pezzola S, D'Aguanno S, Tregno FP, Urbani A, Serra M, Pasello M, Picci P, Federici G and Caccuri AM: Targeting GSTP1-1 induces JNK activation and leads to apoptosis in cisplatin-sensitive and -resistant human osteosarcoma cell lines. Mol Biosyst 8: 994-1006, 2012.

39. Chen B, Liu J, Chang Q, Beezhold K, Lu Y and Chen F: JNK and STAT3 signaling pathways converge on Akt-mediated phosphorylation of EZH2 in bronchial epithelial cells induced by arsenic. Cell Cycle 12: 112-121, 2013.

40. Chen F: JNK-induced apoptosis, compensatory growth, and cancer stem cells. Cancer Res 72: 379-386, 2012

41. Bubici C and Papa S: JNK signalling in cancer: In need of new, smarter therapeutic targets. Br J Pharmacol 171: 24-37, 2014.

42. Seki E, Brenner DA and Karin M: A liver full of JNK: Signaling in regulation of cell function and disease pathogenesis, and clinical approaches. Gastroenterology 143: 307-320, 2012

43. Leventaki V, Drakos E, Medeiros LJ, Lim MS, Elenitoba-Johnson KS, Claret FX and Rassidakis GZ: NPM-ALK oncogenic kinase promotes cell-cycle progression through activation of JNK/cJun signaling in anaplastic large-cell lymphoma. Blood 110: 1621-1630, 2007. 
44. Jia G, Kong R, Ma ZB, Han B, Wang YW, Pan SH, Li YH and Sun B: The activation of c-Jun $\mathrm{NH}_{2}$-terminal kinase is required for dihydroartemisinin-induced autophagy in pancreatic cancer cells. J Exp Clin Cancer Res 33: 8, 2014.

45. Shi Y, Nikulenkov F, Zawacka-Pankau J, Li H, Gabdoulline R, $\mathrm{Xu} \mathrm{J}$, et al: ROS-dependent activation of JNK converts p53 into an efficient inhibitor of oncogenes leading to robust apoptosis. Cell Death Differ 21:612-23, 2014

46. Chen YH, Hao LJ, Hung CP, Chen JW, Leu SF and Huang BM: Apoptotic effect of cisplatin and cordycepin on OC3 human oral cancer cells. Chin J Integr Med 20: 624-632, 2014.

47. Noutomi T, Itoh M, Toyota H, Takada E and Mizuguchi J: Tumor necrosis factor-related apoptosis-inducing ligand induces apoptotic cell death through c-Jun NH2-terminal kinase activation in squamous cell carcinoma cells. Oncol Rep 22: $1169-1172,2009$.

48. Li Q, Song XM, Ji YY, Jiang H and Xu LG: The dual mTORC1 and mTORC2 inhibitor AZD8055 inhibits head and neck squamous cell carcinoma cell growth in vivo and in vitro. Biochem Biophys Res Commun 440: 701-706, 2013.

49. Kim HJ, Chakravarti N, Oridate N, Choe C, Claret FX and Lotan R: N-(4-hydroxyphenyl)retinamide-induced apoptosis triggered by reactive oxygen species is mediated by activation of MAPKs in head and neck squamous carcinoma cells Oncogene 25: 2785-2794, 2006.

50. Sakai T, Banno Y, Kato Y, Nozawa Y and Kawaguchi M: Pepsin-digested bovine lactoferrin induces apoptotic cell death with JNK/SAPK activation in oral cancer cells. J Pharmacol Sci 98: 41-48, 2005

51. Zhao L, Yue P, Lonial S, Khuri FR and Sun SY: The NEDD8-activating enzyme inhibitor, MLN4924, cooperates with TRAIL to augment apoptosis through facilitating c-FLIP degradation in head and neck cancer cells. Mol Cancer Ther 10: 2415-2425, 2011.

52. Zhang S, Wang XL, Gan YH and Li SL: Activation of c-Jun $\mathrm{N}$-terminal kinase is required for mevastatin-induced apoptosis of salivary adenoid cystic carcinoma cells. Anticancer Drugs 21: 678-686, 2010

53. HourMJ,Lee KH,ChenTL,Lee KT,Zhao Y and Lee HZ:Molecular modelling, synthesis, cytotoxicity and anti-tumour mechanisms of 2-aryl-6-substituted quinazolinones as dual-targeted anti-cancer agents. Br J Pharmacol 169: 1574-1586, 2013.
54. Chen HM, Liu CM, Yang H, Chou HY, Chiang CP and Kuo MY: 5 -aminolevulinic acid induce apoptosis via NF- $\mathrm{kB} / \mathrm{JNK}$ pathway in human oral cancer Ca9-22 cells. J Oral Pathol Med 40: 483-489, 2011.

55. Bhattarai G, Lee YH, Lee NH, Lee IK, Yun BS, Hwang PH and Yi HK: Fomitoside-K from Fomitopsis nigra induces apoptosis of human oral squamous cell carcinomas (YD-10B) via mitochondrial signaling pathway. Biol Pharm Bull 35: 1711-1719, 2012

56. Li C and Johnson DE: Bortezomib induces autophagy in head and neck squamous cell carcinoma cells via JNK activation. Cancer Lett 314: 102-107, 2012.

57. Schramek D, Kotsinas A, Meixner A, Wada T, Elling U, Pospisilik JA, Neely GG, Zwick RH, Sigl V, Forni G, et al: The stress kinase MKK 7 couples oncogenic stress to p53 stability and tumor suppression. Nat Genet 43: 212-219, 2011.

58. Dai Y, Guzman ML, Chen S, Wang L, Yeung SK, Pei XY, Dent P, Jordan CT and Grant S: The NF (nuclear factor) $-\kappa B$ inhibitor parthenolide interacts with histone deacetylase inhibitors to induce MKK7/JNK1-dependent apoptosis in human acute myeloid leukaemia cells. Br J Haematol 151: 70-83, 2010.

59. Lui VW, Peyser ND, Ng PK, Hritz J, Zeng Y, Lu Y, Li H, Wang L, Gilbert BR, General IJ, et al: Frequent mutation of receptor protein tyrosine phosphatases provides a mechanism for STAT3 hyperactivation in head and neck cancer. Proc Natl Acad Sci USA 111: 1114-1119, 2014

60. Gkouveris I, Nikitakis N, Karanikou M, Rassidakis G and Sklavounou A: Erk1/2 activation and modulation of STAT3 signaling in oral cancer. Oncol Rep 32: 2175-2182, 2014.

61. Kim JH, Lee SC, Ro J, Kang HS, Kim HS and Yoon S: Jnk signaling pathway-mediated regulation of Stat 3 activation is linked to the development of doxorubicin resistance in cancer cell lines. Biochem Pharmacol 79: 373-380, 2010.

62. Guo W, Wu S, Wang L, Wei X, Liu X, Wang J, Lu Z Hollingshead $M$ and Fang B: Antitumor activity of a novel oncrasin analogue is mediated by JNK activation and STAT3 inhibition. PLoS One 6: e28487, 2011.

63. Liu J, Chen B, Lu Y, Guan Y and Chen F: JNK-dependent Stat3 phosphorylation contributes to Akt activation in response to arsenic exposure. Toxicol Sci 129: 363-371, 2012.

64. Shirakawa T, Kawazoe Y, Tsujikawa T, Jung D, Sato S and Uesugi M: Deactivation of STAT6 through serine 707 phosphorylation by JNK. J Biol Chem 286: 4003-4010, 2011. 